\title{
Diseño de una solución telefónica IP para la empresa Catel S.A.
}

\author{
Luis Giovanny Guerra Almeida
}

Universidad Central del Ecuador

lgga87@gmail.com

Recibido: 01 de septiembre / Aprobado: 30 de noviembre 2017

\section{Resumen}

El presente artículo abarca el diseño de red para brindar el servicio de telefonía IP a la empresa Catel S.A., además muestra los resultados de pruebas con diferentes códecs, las cuales permitieron establecer la mejor comunicación entre los usuarios de la empresa. Se diseñaron dos soluciones de telefonía IP que permiten a la empresa tener un respaldo como central telefónica digital en caso de que la principal presente errores en su funcionamiento, además se solventaron problemas para enlazar a ciertos usuarios que por su localización no tenían acceso a la red cableada. Las pruebas de calidad de servicio efectuadas en la empresa Catel S.A. mostraron como resultado un servicio de voz y datos de alta calidad en una red controlada, lo cual se vio reflejado en la aceptación del personal por el beneficio prestado.

Palabras clave: telefonía IP, códec, diseño de red, central telefónica, ancho de banda.

\section{Abstract}

This article approaches the network design to provide IP telephony service to the company Catel S.A., it also shows calculations of the bandwidth use with different codecs, the results was established improved the communication among the users 
of the company. Two IP telephony solutions were designed in order to provide a backup as a Private Branch Exchange (PBX) in case the main one fails, in addition communication problems were solved connecting users wirelessly because their location did not have access to the wired network. The tests of QoS at the company Catel C.A. showed a high quality of voice and data service in a controlled network. The personal was satisfied with the choosen service.

Keywords: IP telephony, codec, network design, telephone switchboard, bandwidth. 


\section{Introducción}

$\mathrm{L}$

a telefonía desde su invención se ha convertido en uno de los sistemas con mayor aceptación para la comunicación global entre los usuarios, además de ser una herramienta muy importante en el desarrollo de la sociedad. La evolución de la telefonía conjuntamente con el avance de las Tecnologías de la Investigación y Conocimiento (TIC) marcan un punto de partida para la llamada "Era del conocimiento", época actual donde el intercambio de experiencias, sucesos y eventos fomentan el conocimiento e influyen directamente en el desarrollo de la tecnología. En el transcurso del tiempo se han dado un sinnúmero de avances en la telefonía tanto a nivel estructural (equipo físico), como a nivel de distribución de servicios a millones de usuarios. Los caminos que recorre la voz paquetizada a través de la PSTN se describen a continuación en los siguientes procesos y se grafican en la figura1.

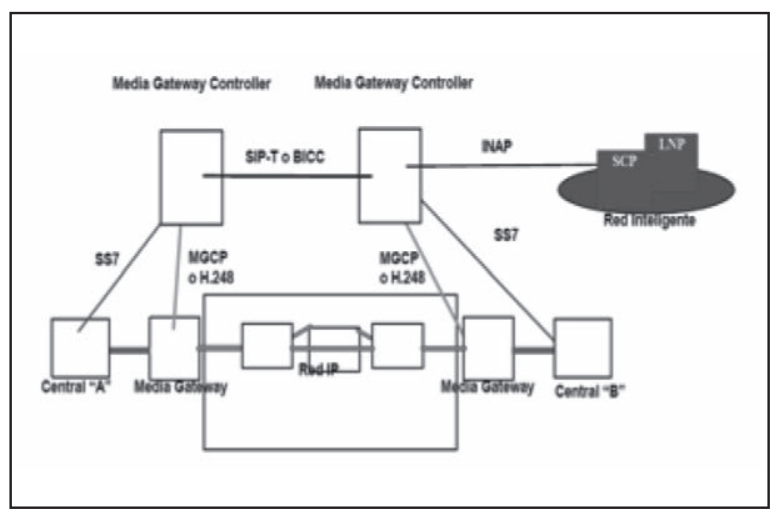

Figura 1. Integración de central VoIP con la PSTN.

La central local se comunica con el "Media Gateway" (MG) a través de tramas E1 ( $2 \mathrm{Mb} / \mathrm{s}$ comunes y corrientes) que transportan la voz viva. El MG es el encargado de traducir las llamadas TDM a paquetes mediante el protocolo RTP (Real Time Protocol) que viaja por la red IP. La separación de funciones es la clave de las redes NGN, en el MG se resuelve el plano de "conectividad" de la red; es decir, el transporte y en el MGC se encuentra el plano de "control”; es decir, la inteligencia. [1]

\section{Protocolos de señalización}

Tienen las siguientes funciones:

- Establecimiento, modificación y finalización de llamadas/sesiones.

- Registro y localización de usuarios.

- Movilidad. 
- Gestión del conjunto de participantes y de los componentes del sistema.

- Descripción de características de las sesiones y negociación de capacidades de los participantes. [2]

\section{Protocolo H.323}

Las operaciones obligatorias que este protocolo debe realizar son las siguientes:

- La traducción de alias o números de teléfono en direcciones IP.

- La administración de ancho de banda y control del tráfico generado por las diferentes comunicaciones, limitando el número máximo de comunicaciones simultáneas.

- Enrutamiento más adecuado para redireccionar la llamada dependiendo de las capacidades del Gateway.

- Control de admisión en la red utilizando para ello mensajes.[3]

\section{Protocolo SIP}

SIP al ser ideado para correr como un servicio más en internet, tiene como característica que su sintaxis es bastante parecida a la que emplea HTTP y SMTP. De una manera más detallada, SIP tiene cinco funciones y son:

- Localización de usuarios.

- Intercambio / negociación de capacidades de los terminales.

- Disponibilidad de usuarios.

- Establecimiento de llamada.

- Mantenimiento de llamada.[4]

\section{Protocolos de transporte}

\section{RTP (Protocolo de transporte en tiempo real)}

Creado por la IETF para la transmisión confiable de voz y video a través de Internet.

En VoIP el protocolo RTP es el responsable de la transmisión de la voz, mientras que la digitalización y compresión de la voz y el vídeo es realizada por el códec.

\section{RTCP (Protocolo de control en tiempo real)}

Es un protocolo de control de los flujos RTP, que permite transportar informaciones básicas de los usuarios.[5]

\section{Códecs}

Al ser la voz una señal analógica y la red de datos una señal digital, necesariamente se requiere un proceso de conversión de ondas análogas a digitales, es decir 
de codificación-decodificación (CODEC), el cual se puede realizar de diversos modos denominados estándares [6]. En la tabla 1 se muestran las características de los principales códec:

Tabla 1. Características de los códec principales

\begin{tabular}{|c|c|c|c|c|}
\hline Códec & Bit Rate & Sampling Rate & Frame Size & MOS \\
\hline G.711 & $64 \mathrm{~kb} / \mathrm{s}$ & $8 \mathrm{kHz}$ & $10 \mathrm{~ms}$ & 4.1 \\
\hline G.729 & $8 \mathrm{~kb} / \mathrm{s}$ & $8 \mathrm{kHz}$ & $10 \mathrm{~ms}$ & 3.92 \\
\hline GSM & $13 \mathrm{~kb} / \mathrm{s}$ & $8 \mathrm{kHz}$ & $22.5 \mathrm{~ms}$ & $3.5-3.9$ \\
\hline iLBC & $8 \mathrm{~kb} / \mathrm{s}$ & $13.3 \mathrm{kHz}$ & $\mathrm{ms}$ & $>\mathrm{G} .729$ \\
\hline
\end{tabular}

\section{Materiales y métodos}

Para cumplimentar los objetivos de garantizar una solución integral de telefonía digital se utilizó la investigación teórica de los protocolos y analizaron las condiciones actuales de la empresa conjuntamente con las prestaciones tecnológicas de las centrales telefónicas, considerando la opción de software libre y la otra de código cerrado.

\section{Elastix}

Es una plataforma de distribución libre que fue creada y es mantenida actualmente por la compañía ecuatoriana PaloSantoSolutions, distribuida bajo licencia GPL versión 2.

Corre sobre el sistema operativo CentOs y se basa en cuatro programas de software que emplean código libre y son: Asterisk, Hylafax, Openfire y Postfix, los cuales integran en un solo paquete: VoIP PBX, fax, mensajería instantánea, correo electrónico y aplicaciones de colaboración [7]. Su estructura se muestra en la figura 2.

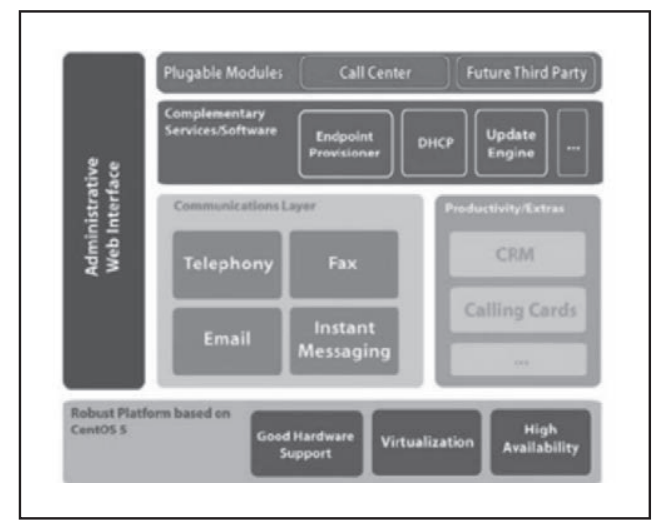

Figura 2. Estructura de Elastix. 


\section{Snom Soho}

La IP PBX Snom en su versión Soho está destinada a solventar los problemas netamente de voz de la empresa. Es un sistema telefónico diseñado específicamente para las pequeñas empresas con hasta 10 extensiones. Ideal para los bufetes de abogados, de médicos o de pequeñas oficinas que necesitan un conjunto de características de solución de comunicaciones a un precio accesible. Es posible ejecutarla y conectarla a la red mediante un servidor que tenga levantadas sus funciones básicas.

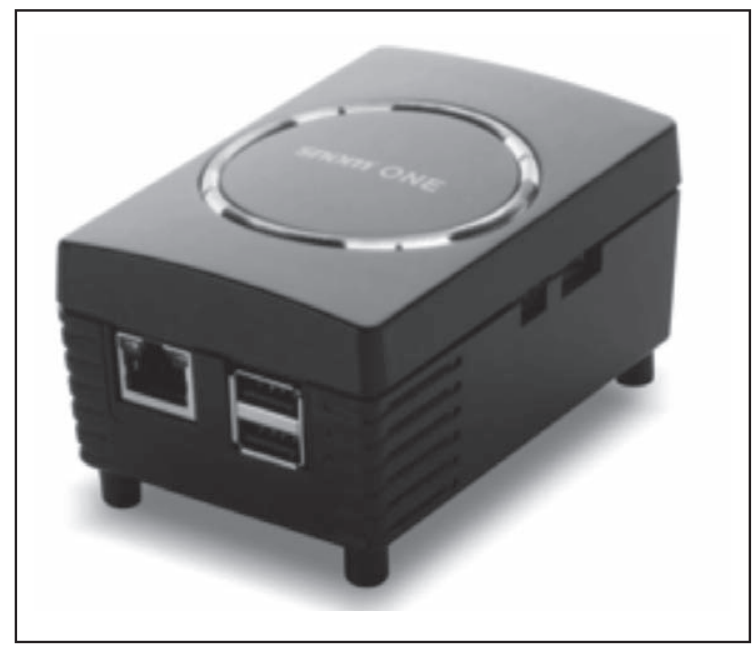

Figura 3. IP PBX Snom Soho.

\section{Toma de decisiones}

Tomando en cuenta las características de las IP-PBX de software gratuito disponibles en internet y los equipos con posibilidad de adquirirse por Catel S.A. se tomaron las siguientes decisiones para brindar una solución integral de telefonía IP:

PBX Primaria: Snom ONE Soho

Las razones fundamentales para elegir a Snom como PBX primaria son:

- $100 \%$ de cumplimiento con los requerimientos de telefonía del presidente.

- Garantía de funcionamiento y estabilidad al tener su hardware encapsulado.

- Bajo consumo de energía.

- Solución económica y atractiva para empresas con pocos empleados.

PBX Backup: Elastix

Es una PBX de software libre con una amplia gama de funcionalidades; en nuestro caso se hallaron las siguientes desventajas: 
- Desenvolvimiento condicionado al correcto funcionamiento de una máquina virtual donde se monte esta PBX.

- Compartición de la interfaz de red del servidor.

- PBX VoIP en ordenador con memoria RAM limitada y con otros servicios corriendo simultáneamente.

En base a los estudios realizados, se puede determinar que ninguno de los tres códec afecta a las comunicaciones web o de telefonía en la empresa. La demanda de telefonía es irregular en la empresa, es decir nunca se presenta consumo simultáneo de las cuatro troncales, por tal motivo se decidió configurar la PBX y los terminales IP bajo el códec G.711 que es el cual ofrece la mayor calidad de voz según el MOS, debido a sus niveles de cuantización y una mayor tasa de transmisión de voz en sus paquetes.

\section{Levantamiento de información}

Requerimiento de ancho de banda para internet

Para realizar este estudio se considerará que un usuario puede acceder a 6 páginas web en una hora cuyo tamaño promedio es de $507 \mathrm{~KB}^{1}$, entonces se debe aplicar el cálculo de throughput resultante por la navegación de este usuario:

$$
\begin{gathered}
\operatorname{Trp}_{w} * \text { usuario }=\frac{507 \mathrm{~KB}}{\text { página }} * \frac{8 \text { bits }}{\text { Byte }} * \frac{6 \text { páginas }}{3600 \mathrm{seg}}=6,76 \mathrm{Kbps} \\
\operatorname{Trp}_{w} \text { total }=6,76 \mathrm{Kbps} * 6 \text { usuarios }=40,56 \mathrm{Kbps}
\end{gathered}
$$

Si se considera que un usuario puede leer 3 correos electrónicos por hora, cuyo tamaño máximo es de $50 \mathrm{~KB}^{2}$, se puede determinar la tasa promedio que genera el consumo de correo electrónico:

$$
\begin{gathered}
\operatorname{Trp}_{c} * \text { usuario }=\frac{50 \mathrm{~KB}}{\text { correo }} * \frac{8 \text { bits }}{\text { Byte }} * \frac{3 \text { correos }}{3600 \mathrm{seg}}=0,33 \mathrm{Kbps} \\
\operatorname{Trp}_{c} \text { total }=0,33 \mathrm{Kbps} * 6 \text { usuarios }=1,98 \mathrm{Kbps}
\end{gathered}
$$

Entonces se puede determinar la velocidad de transmisión que los usuarios de la empresa requieren para contar con todos los servicios de internet.

$$
\begin{gathered}
\operatorname{Tr} p_{\text {isp }} \text { total }=\operatorname{Tr}_{w} \text { total }+\operatorname{Tr}_{c} \text { total }=40,56 \mathrm{Kbps}+1,98 \mathrm{Kbps} \\
\operatorname{Tr} p_{i s p} \text { total }=42,54 \mathrm{Kbps}
\end{gathered}
$$

1 Cambiar el tamaño máximo del correo electrónico, http://www.shareo.com/es/WebHelp/Change_max_size_data_emails.htm

2 Hurtado, Roberth, "Diseńo de la red inalámbrica integrada de voz y datos con calidad de servicio y seguridades de red para la Casa Matriz del Banco Nacional de Fomento”, Quito, mayo, 2008. 
Como se puede apreciar el ancho de banda disponible en la empresa es suficiente para la demanda de los servicios web o requerimientos de internet.

Requerimientos para servicio de VoIP y conexión remota a la PBX

La lista de funcionalidades que el presidente de Catel S.A. solicitó para posteriormente determinar la PBX más apta y económica se muestra a continuación:

- Detector de llamada

- Acceso por control de contraseñas a usuario y administrador

- Operadora automática

- Hermanamiento con teléfono móvil

- Grabación de llamadas

- Restricción de llamadas

- Filtrado de llamadas

- Registros múltiples por extensión

- Múltiples troncales por dominio

- Correo de voz a correo electrónico

- Detección de entradas DTMF

- Lista negra

- Calidad de servicio (QoS)

En cuanto a demanda de servicios como mensajería instantánea o correo electrónico interno, el presidente supo manifestar que eran servicios irrelevantes ya que el ISP provee este servicio mediante un dominio de correo electrónico a la empresa y la mensajería instantánea no era necesaria.

Adicionalmente, se solicitó como requerimiento, que se tenga conectividad desde cualquier lugar en el mundo a la central telefónica digital que se decida implementar, es decir que el internet sea un medio de conexión con la PBX. Se dieron a conocer las razones de este requerimiento por parte del presidente, la principal razón es que el gerente realiza continuamente viajes al exterior y es de vital importancia para Catel S.A. que los procesos y proyectos involucrados sigan en pie, por tal motivo la comunicación entre personal administrativo, presidente y el gerente es primordial.

\section{Levantamiento de información}

Para ofrecer una solución integral de telefonía en la empresa fue necesario diseñar una topología lógica que involucre los equipos dedicados a suplir los servicios de datos (Internet), Voz sobre IP, intercomunicación con la Red de Servicio de Telefonía Pública (PSTN). El plano de la empresa se muestra en la figura 4, cuya distribución cableada e inalámbrica está graficada en la figura 5. 
Determinación del mejor códec para VoIP.

Se debe hacer un estudio basándonos en el ancho de banda que consumen los códec a implementarse, además se plantea un ambiente de total saturamiento de las líneas telefónicas para evitar cualquier inconveniente por un insuficiente ancho de banda.

$\mathrm{Al}$ existir cuatro troncales lógicas disponibles en la empresa, se realizan los cálculos de 4 usuarios realizando llamadas simultáneamente utilizando cada canal en sentido full-dúplex, empleando la siguiente fórmula:

Tamańo total del paquete $=$ Cabecera en L2 o Ethernet + Cabecera en L3IP/ UDP/RTP + Tamaño de carga útil de voz (Payload)

Paquetes por segundo (PPS)

$$
\begin{aligned}
& \mathrm{PPS}=\frac{\text { tasa de bits del códec }(\text { codec bitrate })}{\text { tamaño carga útil de voz (voice payload size })(\text { bits })} \\
& \boldsymbol{B} \boldsymbol{W}_{\text {por llamada }}=\text { Tamaño total PKT } * \mathrm{PPS}^{1}[\mathrm{kbps}]
\end{aligned}
$$

\section{Resultados}

Luego de comprender la fundamentación teórica para obtener la tasa real de transmisión de datos + voz se realizaron los cálculos respectivos, los cuales se describen a continuación:

Para realizar los cálculos se asumieron ciertos valores:

- El tamaño de la cabecera L2 es 18 bytes de Ethernet, incluyendo [CRC] o [FCS]

- La cabecera L3 es de 40 bytes [IP (20 bytes)+ UDP ( 8 bytes)+ RTP (12 bytes)] Conocidas las variables que se necesitan para determinar el consumo de ancho de banda por llamada se genera la tabla 2 con la información requerida:

Tabla 2. Información necesaria de códec

\begin{tabular}{|c|c|c|c|}
\hline Códec & G.711 & G.729 & GSM 6.10 \\
\hline $\begin{array}{c}\text { Cabeceras } \\
\text { L2 + L3 [Bytes] }\end{array}$ & 58 & 58 & 58 \\
\hline Payload [Bytes] & 160 & 20 & 33 \\
\hline Tamańo del paquete [bits] & 1744 & 624 & 728 \\
\hline Bitrate del códec [kbps] & 64 & 8 & 13 \\
\hline Payload [bits] & 1280 & 160 & 264 \\
\hline PPS & 50 & 50 & 49,24 \\
\hline
\end{tabular}


Al ser cuatro llamadas simultáneas y en sentido full dúplex es necesaria realizar la siguiente operación:

Los valores de un ambiente saturado de llamadas VoIP se muestran en la tabla 3.

Tabla 3. Consumo de ancho de banda para VoIP

\begin{tabular}{|c|c|c|}
\hline Códec & {$[\mathbf{k b p s}]$} & {$[\mathbf{k b p s}]$} \\
\hline G.711 & 87,2 & 697,6 \\
\hline G.729 & 31,2 & 249,6 \\
\hline GSM 6.10 & 35,85 & 286,77 \\
\hline
\end{tabular}

Finalmente se debe simular el consumo de telefonía digital y servicios web simultáneamente. Estos valores se muestran en la tabla 4.

Tabla 4. Consumo total de ancho de banda

\begin{tabular}{|c|c|c|c|}
\hline Códec & {$[\mathbf{k b p s}]$} & {$[\mathbf{k b p s}]$} & {$[\mathbf{k b p s}]$} \\
\hline G.711 & 697,2 & 42,54 & 739,74 \\
\hline G.729 & 249,6 & 42,54 & 292,14 \\
\hline GSM 6.10 & 286,77 & 42,54 & 329,31 \\
\hline
\end{tabular}

\section{Diseño de red}

Es primordial realizar un diseño de acuerdo a la disponibilidad de los equipos con los que cuenta la empresa, y estudiar detenidamente el cableado o soluciones inalámbricas que se deben implementar para solventar cualquier problema que se presente. Inicialmente la red empresarial tenía distribuidos los equipos como se muestran en la figura 4.

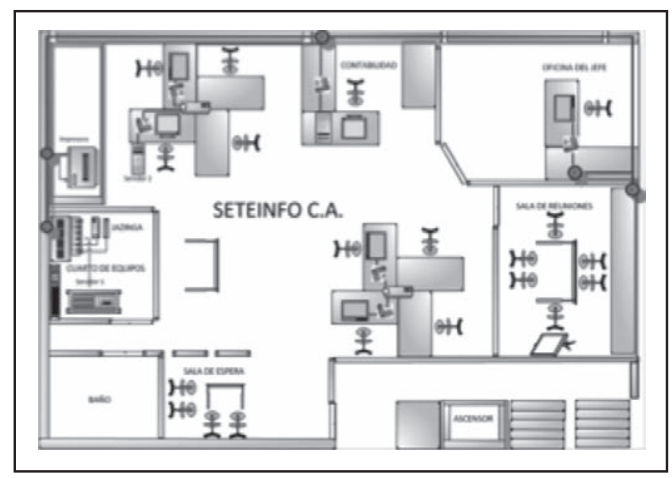

Figura 4. Planos de la empresa y cableado inicial 
Se empezó a desarrollar el diagrama lógico mostrado en la figura 5, de acuerdo a los requerimientos solicitados por la empresa, es decir implementar dos centrales telefónicas digitales, una de tecnología Snom y otra basada en software libre, además de permitir conectividad remota desde los usuarios de una red sucursal y desde usuarios ubicados remotamente.

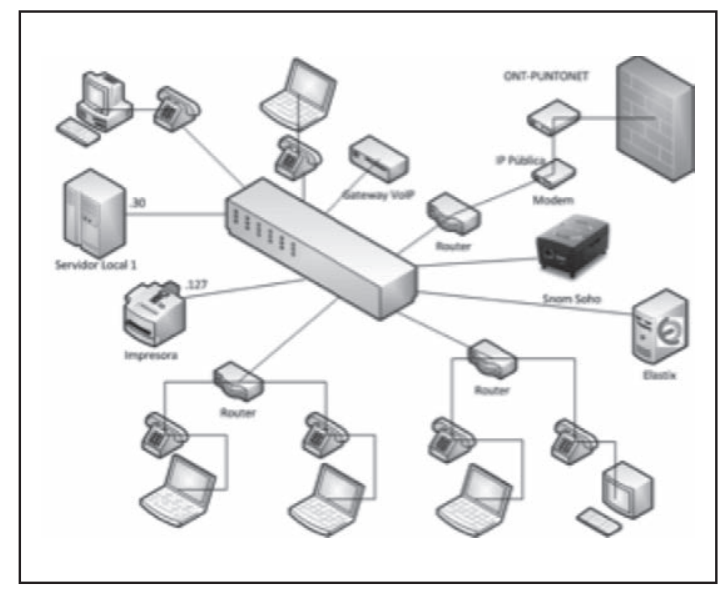

Figura 5. Diagrama lógico inicial.

Como se pudo apreciar en las figuras 4 y 5 , todos los equipos mantienen una conexión alámbrica y se solicitó como requerimiento que se comuniquen inalámbricamente a las islas con la red empresarial, es decir que se configuren los routers ubicados en las islas en modo Access Point.

Siendo acogido este requerimiento tuvo que modificarse el diagrama lógico inicial a una versión definitiva mostrada en la figura 6.

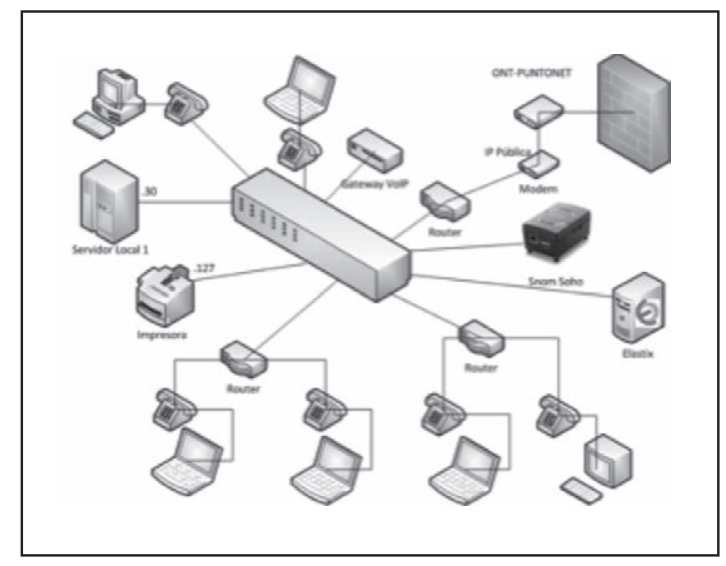

Figura 6. Diagrama lógico final. 


\section{Configuración de los equipos}

Se realizaron las respectivas configuraciones en los diferentes equipos de red que se adquirieron y con los que contaba la empresa, siendo las principales las que se enumeran a continuación:

\section{Routers}

- Actualización de firmware

- Conectividad wireless (canales y seguridad)

- Conectividad a la WAN

- Conectividad a la LAN

- Creación y registro de dominios dinámico para la empresa

- Configuración de servicio para redes privadas virtuales

- Enrutamiento estático entre routers

En cuanto a conectividad con las islas en las instalaciones de la empresa se debieron realizar los siguientes procedimientos:

Router emisor - Interfaz inalámbrica virtual y registro de MAC’s para repetidores.

Routers repetidores - Autenticación en red inalámbrica virtual, con el mismo canal y uso del mismo, además autenticación con el router emisor por MAC.

PBX Snom Soho y Elastix

- Configuración de IP estática

- Configuraciones generales (extensiones, dominios, planes de marcado, IVR, restricciones, etc.)

- Configuración de troncales

En el caso de Elastix se levantaron los servicios de mensajería instantánea y correo electrónico.

Gateway VoIP

- Configuración de red

- Parámetros generales SIP (configuración de registro y proxy, prioridad de los codecs, registro de troncales, marcados DTMF, etc.)

Teléfonos IP y softphones

- Configuración de IP estática

- Compatibilidad de códigos de funcionalidades para cada IP PBX 


\section{Conclusiones}

- Se diseñó e implementó una solución integral de telefonía IP en la empresa Catel S.A. con la utilización de la técnica de voz sobre IP ofreciendo una alta calidad de servicio.

- Se intercomunicaron a las redes local y remota permitiendo tener a los usuarios de la empresa Catel S.A. intercomunicados y contando con las bondades que la tecnología Snom ofrece a sus clientes.

- Fue diseñada e implementada Elastix como una central telefónica digital redundante basada en software libre para poder realizar un estudio comparativo entre las dos tecnologías.

- Se optimizó la red de la empresa implementando routers repetidores que permitieron la conectividad de usuarios que no tenían acceso a nodos cableados de conexión a la red interna.

- Se documentó todo el proceso de instalación y configuración del software: Snom, Elastix, ddwrt y AudioCodes, así como la implementación de su hardware en la empresa Catel S.A.

- Se realizó un manual de usuario que permite al personal de la empresa Catel S.A. tener conocimientos básicos del funcionamiento de las centrales telefónicas y solventar cualquier inconveniente en el uso cotidiano de los terminales basados en telefonía sobre IP.

\section{Referencias}

[1] Telefónica. "Entendiendo la tecnología VoIP”. Contribución técnica.

[2] Moreno, José Soto, Ignacio Larrabeiti, David. "Protocolos de señalización para el transporte de voz sobre redes IP”. Universidad de Madrid.

[3] VoIP, Protocolo H.323, http://www.redesyseguridad.es/voip-protocolo-h323

[4] Montesino, Federico. "SIP: Session Initiation Protocol", XV Grupos de Trabajo de Red IRIS, mayo 2003.

[5] "RTP y RTCP". Etudes et formations en télécommunications (EFORT).

[6] Codecs, http://www.voipforo.com/codec/codecs.php, 10 de abril/2012.

[7] Landívar, Edgar. Comunicaciones unificadas con Elastix, Volumen 1.

[8] DITG (Data Inyector Traffic Generator), http://www.grid.unina.it/software/ITG/ 